\title{
Global Stable Disease
}

National Cancer Institute

\section{Source}

National Cancer Institute. Global Stable Disease. NCI Thesaurus. Code C159948.

Failure to attain complete response, partial response, or progressive disease

representative of all disease. Partial response in skin and no nodes, blood and viscera

category has a progressive disease and if any category involved at baseline, no complete or partial response in any. 